\title{
An Open-Label, 8-Week Study of Safety and Efficacy of Pimavanserin Treatment in Adults with Parkinson's Disease and Depression
}

\author{
Daryl DeKarske ${ }^{\mathrm{a}, *}$, Gustavo Alva ${ }^{\mathrm{b}}$, Jason L. Aldred ${ }^{\mathrm{c}}$, Bruce Coate ${ }^{\mathrm{a}}$, Marc Cantillon ${ }^{\mathrm{d}}$, Lori Jacobi ${ }^{\mathrm{a}}$, \\ Rene Nunez ${ }^{\mathrm{a}}$, James C. Norton ${ }^{\mathrm{a}, 1}$ and Victor Abler ${ }^{\mathrm{a}}$ \\ ${ }^{a}$ ACADIA Pharmaceuticals Inc., San Diego, CA, USA \\ ${ }^{\mathrm{b}}$ Department of Neuroscience, University of California, Riverside, Riverside, CA, USA \\ ${ }^{\mathrm{c} S}$ Selkirk Neurology, PLLC, Spokane, WA, USA \\ ${ }^{\mathrm{d}}$ Department of Neurology and Psychiatry, Robert Wood Johnson Medical School, New Brunswick, NJ, USA
}

Accepted 17 July 2020

\begin{abstract}
.
Background: Many patients with Parkinson's disease (PD) experience depression.

Objective: Evaluate pimavanserin treatment for depression in patients with PD.

Methods: Pimavanserin was administered as monotherapy or adjunctive therapy to a selective serotonin reuptake inhibitor or serotonin/noradrenaline reuptake inhibitor in this 8-week, single-arm, open-label phase 2 study (NCT03482882). The primary endpoint was change from baseline to week 8 in Hamilton Depression Scale-17-item version (HAMD-17) score. Safety, including collection of adverse events and the Mini-Mental State Examination (MMSE) and Movement Disorder Society-sponsored revision of the Unified Parkinson's Disease Rating Scale Part III (MDS-UPDRS III) scores, was assessed in patients who received $\geq 1$ pimavanserin dose.

Results: Efficacy was evaluated in 45 patients (21 monotherapy, 24 adjunctive therapy). Mean (SE) baseline HAMD-17 was 19.2 (3.1). Change from baseline to week 8 (least squares [LS] mean [SE]) in the HAMD-17 was -10.8 (0.63) (95\% CI, -12.0 to $-9.5 ; p<0.0001)$ with significant improvement seen at week $2(p<0.0001)$ and for both monotherapy (week 8 , $-11.2[0.99]$ ) and adjunctive therapy (week $8,-10.2$ [0.78]). Most patients $(60.0 \%)$ had $\geq 50 \%$ improvement at week 8 , and $44.4 \%$ of patients reached remission (HAMD-17 score $\leq 7$ ). Twenty-one of 47 patients experienced 42 treatment-emergent adverse events; the most common by system organ class were gastrointestinal $(n=7 ; 14.9 \%)$ and psychiatric $(n=7 ; 14.9 \%)$. No negative effects were observed on MMSE or MDS-UPDRS Part III.

Conclusion: In this 8-week, single-arm, open-label study, pimavanserin as monotherapy or adjunctive therapy was well tolerated and associated with early and sustained improvement of depressive symptoms in patients with PD.
\end{abstract}

Keywords: Parkinson's disease, depression, dementia, pimavanserin, adjunctive therapy, monotherapy

\footnotetext{
${ }^{1}$ Current affiliation is Arena Pharmaceuticals, San Diego, CA, USA

*Correspondence to: Daryl DeKarske, MPH, ACADIA Pharmaceuticals Inc., 3611 Valley Centre Drive, Ste 300, San Diego,
}

CA 92130-3331, USA. Tel.: +1 858320 8683; $\quad$ E-mail: ddekarske@acadia-pharm.com. 


\section{INTRODUCTION}

Parkinson's disease (PD) is a progressive neurodegenerative disorder characterized primarily by motor deficits; however, behavioral symptoms associated with the disease are frequent, severe, and disabling [1]. Mood disorders, including depression, occur in $30 \%$ to $50 \%$ of patients $[1,2]$. Depression can emerge at any phase of the disease [1] and is associated with faster progression of physical symptoms, disability, and diminished quality of life [3]. Conversely, improvement of depression in patients with PD correlates with reduced physical disability and improved quality of life [3].

Despite the significant need for treatment, no medications are currently approved by the United States Food and Drug Administration (US FDA) for depression in patients with PD. A paucity of studies have examined currently approved antidepressants for treating depression in patients with PD [4-7] and suggest either no benefit or only marginal effects.

Widespread neurodegeneration and monoaminergic dysregulation, particularly in the mesolimbic system, can contribute to depression [8,9]. While the exact pathophysiology is unknown, key features of depression, including depressed mood, apathy, and anhedonia, are known to be related to serotonergic transmission that is dysregulated in PD [9-11]. Compounds with potent antagonist/inverse agonist activity at 5-hydroxytryptamine $2 \mathrm{~A}\left(5-\mathrm{HT}_{2 \mathrm{~A}}\right)$ receptors, and to varying degrees at 5-hydroxytryptamine $2 \mathrm{C}\left(5-\mathrm{HT}_{2} \mathrm{C}\right)$ receptors, but with lower affinity for monoamine transporters, have shown antidepressant effects in major depressive disorder patient populations $[12,13]$.

Pimavanserin is a selective $5-\mathrm{HT}_{2 \mathrm{~A}}$ receptor antagonist/inverse agonist with limited affinity for $5-\mathrm{HT}_{2 \mathrm{C}}$ receptors [14]. Based on its mechanism of action, pimavanserin may have antidepressant activity. In a phase 2 randomized, controlled trial, adjunctive pimavanserin treatment improved symptoms of major depressive disorder (MDD) in patients with an inadequate response to selective serotonin reuptake inhibitor (SSRI) or serotonin/norepinephrine reuptake inhibitor (SNRI) treatment [15]. The current study was intended to preliminarily assess the efficacy and safety of pimavanserin being investigated for the treatment of depression in patients with PD.

\section{METHODS}

\section{Study design}

This was an 8-week, open-label, single-arm phase 2 study to evaluate the efficacy and safety of pimavanserin for treatment of depression in patients with PD. During the study, patients were given pimavanserin $34 \mathrm{mg}$ (two $17 \mathrm{mg}$ tablets) with instructions to take the medication orally at approximately the same time daily. Assessments were conducted every 2 weeks, and patients received a safety followup call 2 weeks after the last dose of pimavanserin (Fig. 1).

The study was conducted between March 9, 2018, and July 24, 2019 (ClinicalTrials.gov identifier: NCT03482882). All procedures were conducted in accordance with the Declaration of Helsinki and applicable International Council for Harmonisation of Technical Requirements for Pharmaceuticals for Human Use and Good Clinical Practice guidelines. The protocol was approved by each site's ethics committee or institutional review board and all participants provided informed consent.

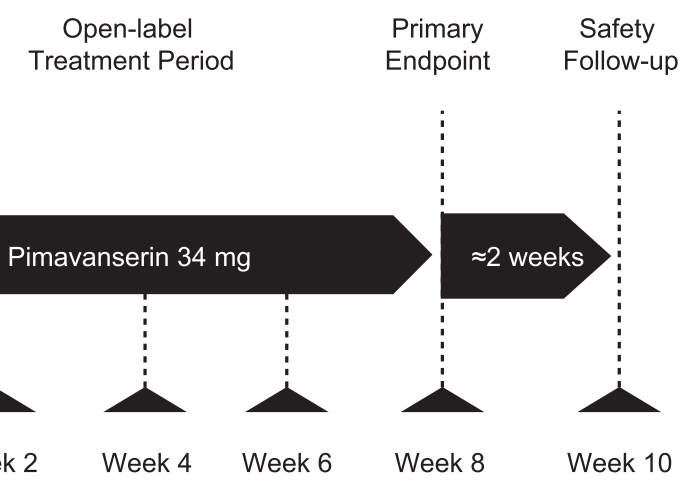

Fig. 1. Study design. PD, Parkinson's disease; SNRI, serotonin/norepinephrine reuptake inhibitor; SSRI, selective serotonin reuptake inhibitor. 


\section{Patient eligibility}

Patients in the United States aged $\geq 50$ years with $\mathrm{PD}$ and depression [16] were recruited. Patients were required to have a clinical diagnosis of $\mathrm{PD}$ for $\geq 1$ year with $\geq 3$ symptoms of $\mathrm{PD}$ (rest tremor, rigidity, bradykinesia or akinesia, or postural/gait abnormalities), be on anti-PD medication for $\geq 1$ year, and exhibit a Mini-Mental State Examination (MMSE [17]) score of $\geq 21$.

PD patients with depressive symptoms (score of $\geq 15$ on the Hamilton Depression Scale-17item version (HAMD-17 [18]) and who met the National Institute of Neurological Disorders and Stroke-National Institute of Mental Health criteria for depression in patients with PD [16]) were eligible to enroll. Those taking a single SSRI or SNRI within the US FDA approved labeling for the treatment of major depressive disorder were eligible if their current treatment was inadequate. If patients were taking more than one antidepressant, they were eligible to enroll if they were being discontinued from one agent before the baseline visit in a clinically appropriate manner.

Patients were excluded if they were taking or had taken an antipsychotic medication (within 3 weeks or 5 half-lives of the baseline visit [whichever is longer]); had a history of PD psychosis, schizophrenia, bipolar I or II disorder, another psychotic disorder, or substance use disorder (within the last 6 months); or were actively suicidal. Individuals with a history of stroke, a family history of a long QT syndrome, or a myocardial infarction were also excluded. Full inclusion/exclusion criteria are listed in Supplementary Table 1. Adjunctive medications for Parkinson's disease or depression were kept stable for the duration of the study if possible.

\section{Outcomes and assessments}

The primary objective of the study was to assess the efficacy of pimavanserin treatment, as monotherapy or adjunctive therapy, in improving depression symptoms in adults with PD. The primary endpoint was assessed based on the change from baseline to week 8 in depression symptoms as measured by the HAMD-17 [18]. The proportion of patients showing $\geq 50 \%$ improvement in the HAMD-17 score was evaluated as a secondary endpoint. A post hoc analysis examined the proportion of patients who reached remission, defined as a HAMD-17 score of $\leq 7$ [19]. The HAMD-17 was administered at screening, baseline, and weeks 2, 4, 6, and 8 .
Secondary endpoints evaluated the effect of pimavanserin treatment on clinicians' global assessment of illness, sleep quality, and overall quality of life. Global impression of illness was assessed by the Clinical Global Impression-Improvement (CGI-I) score and change from baseline on the Clinical Global Impression-Severity (CGI-S) score [20]. Changes in sleep quality were evaluated as change from baseline in the Scale for Outcomes of PD-Sleep Scale (SCOPA) score, including global sleep (GS) quality, nighttime sleep (NS) quality, and daytime sleepiness (DS) [21]. Patients' quality of life was assessed by a caregiver as change from baseline in the EuroQol-5 Dimensions-5 Levels Proxy version 1 visual analog scale (EQ-5D-5L-VAS) [22]. The CGI-S and CGI-I were assessed at baseline and weeks 2, 4, 6, and 8 . SCOPA and EQ-5D-5L-VAS were assessed at baseline and at weeks 4 and 8 .

Medical history and demographic information were collected during screening. The MMSE [17] and Columbia-Suicide Severity Rating Scale (C-SSRS) scores [23] were used to screen for cognitive impairment and suicidal ideation. Both measures were also collected as safety assessments. The MMSE was administered at screening, baseline, week 4, and week 8. The C-SSRS was administered at screening, baseline, and weeks 2, 4, 6, and 8 . The Massachusetts General Hospital Antidepressant Treatment Response Questionnaire (MGH ATRQ) $[24,25]$ was used as a screening assessment. The MGH ATRQ assesses response to antidepressant treatment retrospectively using specific anchor points throughout treatment history to define the adequacy of the dose and duration of each antidepressant course. Patients exhibiting a maximum improvement of $<75 \%$ with an existing antidepressant were included in the adjunctive therapy group.

Safety endpoints, including the MMSE, C-SSRS, physical examinations, vital signs, clinical laboratory tests, electrocardiograms, and incidence of adverse events (AEs) were monitored throughout the study. A treatment-emergent AE (TEAE) was defined as an $\mathrm{AE}$ with onset date during treatment or within 30 days of the last study drug dose date. The relationship to treatment was reported by the investigator. A serious $\mathrm{AE}$ was defined as an $\mathrm{AE}$ that was fatal, was immediately life-threatening, resulted in disability or permanent damage, required hospitalization, was a congenital anomaly or birth defect in an offspring, or was medically significant.

The Movement Disorder Society (MDS)sponsored revision of the Unified Parkinson's Dis- 
ease Rating Scale (UPDRS) Part III (MDS-UPDRS III) [26], a comprehensive measure of motor indices, was also included to assess any decline in motor function. This assessment was completed in the "on" state and was conducted at baseline, week 4, and week 8; change from baseline was analyzed.

\section{Statistical analysis}

Patients who received $\geq 1$ dose of pimavanserin and completed a baseline and $\geq 1$ postbaseline HAMD-17 assessment were included in the efficacy analysis. The mixed model repeated measures (MMRM) method was used to analyze primary and secondary endpoints. The total score of each measure was analyzed, with change from baseline as the dependent variable and baseline total score (of the dependent variable), visit (weeks $2,4,6$, and 8 ), and base-

Table 1

Baseline demographics and disease characteristics

\begin{tabular}{lc}
\hline Characteristic & $\mathrm{N}=45$ \\
\hline Age, $\mathrm{y}$ & \\
$\quad$ Mean (SD) & $69.3(8.3)$ \\
Range & $51-89$ \\
Age category, $\mathrm{n}(\%)$ & \\
$<$ <0 & $20(44.4)$ \\
$\geq 70$ & $25(55.6)$ \\
Male, $\mathrm{n}(\%)$ & $23(51.1)$ \\
Female, $\mathrm{n}(\%)$ & $22(48.9)$ \\
Race, $\mathrm{n}(\%)$ & \\
White & $41(91.1)$ \\
Black or African American & $2(4.4)$ \\
Asian & $1(2.2)$ \\
Other & $1(2.2)$ \\
Age at PD onset, mean (SD), y & $61.5(9.9)$ \\
Duration of PD, mean (SD), y & $7.8(5.5)$ \\
Age at onset of depression with PD (SD), y & $63.9(10.9)$ \\
Duration of depression with PD (SD), y & $5.4(6.8)$ \\
MMSE score, mean (SD) & $27.6(2.5)$ \\
MDS-UPDRS Part III score, mean (SD) & $30.5(17.3)$ \\
Assessment score, mean (SD) & \\
HAMD-17 & $19.2(3.1)$ \\
CGI-S & $4.1(0.5)$ \\
SCOPA-NS & $6.1(3.4)$ \\
SCOPA-DS & $5.2(3.7)$ \\
SCOPA-GS & $3.9(1.5)$ \\
EQ-5D-5L-VAS & $63.9(16.3)$ \\
\hline
\end{tabular}

CGI-S, Clinical Global Impression-Severity; HAMD-17, Hamilton Depression Scale-17-item version; MDS-UPDRS, Movement Disorder Society-sponsored revision of the Unified Parkinson's Disease Rating Scale; MMSE, Mini-Mental State Examination; PD, Parkinson's disease; SCOPA-DS, Scale for Outcomes in Parkinson's Disease-daytime sleepiness; SCOPA-GS, Scale for Outcomes in Parkinson's Disease-global sleep; SCOPA-NS, Scale for Outcomes in Parkinson's Disease-nighttime sleep; EQ-5D5L-VAS, EuroQol-5 Dimensions-5 Levels Proxy version 1 visual analog scale; SD, standard deviation. line total score-by-visit interaction as independent variables. Treatment effects were reported as the least squares (LS) mean (standard error [SE]) change from baseline to week 8 . The CGI-I was analyzed using the MMRM with CGI-I score as the dependent variable, and with independent variables of baseline CGI-S score, visit, and baseline CGI-S score-by-visit interaction. Significance level was set to 0.05 and $p$ values reported for pre-planned secondary outcomes were unadjusted for multiple comparisons.

The proportion of patients showing a response to treatment was reported by visit. Responders were defined as patients who exhibited $\geq 50 \%$ reduction from baseline score in the HAMD-17. Observed cases (patients with missing values at a given visit were excluded) and missing values imputed as nonresponders were included.

In a post hoc analysis, the proportion of patients reaching remission, defined as a HAMD-17 score $\leq 7$, was reported by visit. Missing values were imputed as nonremitters. Improvement on individual items of the HAMD-17 was also analyzed post hoc using an MMRM similar to the primary endpoint.

Patients who received $\geq 1$ dose of pimavanserin were included in the safety analysis. Safety endpoints were summarized using descriptive statistics. The MMSE and MDS-UPDRS Part III were analyzed using an MMRM similar to the primary endpoint.

\section{RESULTS}

\section{Patients}

Forty-seven patients were enrolled in the study from 14 sites in the United States. Forty-five patients were included in the efficacy analyses. Patients had a mean (standard deviation [SD]) age at baseline of 69.3 (8.3) years and a mean (SD) MMSE score of 27.6 (2.5). The mean (SD) time since PD diagnosis and duration of depression with PD diagnoses was 7.8 (5.5) years and 5.4 (6.8) years, respectively (Table 1). At baseline, patients were "Moderately Depressed" on average, with a mean (SD) HAMD-17 score of 19.2 (3.1), and a range of 15 ("Mild Depression") to 27 ("Severe Depression") [19]. The mean (SD) time since the first use of an antidepressant was 5.4 (5.9) years. Forty patients completed the study and 7 (14.9\%) discontinued. Reasons for discontinuation were adverse event $(n=3,6.4 \%)$, protocol violation $(n=2,4.3 \%)$, loss to follow up $(n=1,2.1 \%)$, or other event (medical monitor decision, $n=1,2.1 \%$ ). 
Twenty-six enrolled patients were administered pimavanserin as adjunctive therapy with one of the following: bupropion, duloxetine, escitalopram, fluoxetine, paroxetine, sertraline, or vortioxetine. Dosage and number of patients taking each are presented in Supplementary Table 2. Twenty-four patients $(53.3 \%)$ taking pimavanserin as adjunctive therapy were included in the primary efficacy analysis. The MGH ATRQ revealed that 14 (58.3\%) of 24 patients included in the adjunctive therapy group reported, a maximum improvement $<50 \%$ with their existing antidepressant medication at screening and $10(41.7 \%)$ of 24 patients reported a maximum improvement of $50 \%$ to $<75 \%$. Patients had been taking antidepressant medication for a minimum of 3 months, and the mean (SE) duration of antidepressant use was 49.0 (12.68) months. For those who discontinued a second antidepressant, the second antidepressant was discontinued within the 21-day screening period prior to the baseline visit. In patients in the adjunctive therapy group, the baseline mean (SD) HAMD-17 score was 19.2 (3.8), with a range of 15 to 27.

Twenty-one patients $(46.7 \%)$ who received pimavanserin as monotherapy were included in the primary efficacy analysis. In these patients, baseline mean (SD) HAMD-17 score was 19.1 (2.1), with a range of 16 to 23 .

\section{Changes in depressive symptoms in PD}

In the primary analysis, which included both monotherapy and adjunctive therapy, patients showed a significant improvement in depression symptoms, as indicated by a significant reduction in the HAMD17 score at week 8 (LS mean [SE] change, -10.8 [0.63]; $95 \% \mathrm{CI},-12.0$ to $-9.5 ; p<0.0001)$, with significant improvement seen as early as week $2(-7.3$ [0.85]; $95 \% \mathrm{CI},-9.0$ to $-5.6 ; p<0.0001$ ) (Fig. $2 \mathrm{~A}$ ). Patients showed significant improvement on all 17 individual items of the HAMD-17 in the post hoc analysis (Fig. 2B).

Patients receiving pimavanserin as monotherapy showed significant improvement in depression symptoms, as indicated by a reduction at week 8 in the HAMD-17 (LS mean [SE] change, -11.2 [0.99]; $95 \%$ CI, -13.3 to $-9.1 ; p<0.0001)$. Again, significant improvements were seen starting at week 2 (Fig. 3A). Patients receiving pimavanserin as adjunctive therapy also exhibited a significant reduction in HAMD-17 score at week 8 (LS mean [SE] change, -10.2 [0.78];
95\% CI, -11.8 to $-8.6 ; p<0.0001)$, with significant improvement starting at week 2 (Fig. 3B).

By week 2, 16 of 45 patients $(35.6 \%$; 95\% CI, 23.2 to 50.2) showed a response to treatment (HAMD17 improvement $\geq 50 \%$ from baseline). This level of improvement was observed in 22 patients $(48.9 \%$; $95 \%$ CI, 35.0 to 63.0$)$ at week 4,25 patients $(55.6 \%$; $95 \% \mathrm{CI}, 41.2$ to 69.1 ) at week 6 , and 27 patients (60.0\%; 95\% CI, 45.5 to 73.0$)$ at week 8. Remission (HAMD-17 score $\leq 7$ ) was achieved by 10 of 45 patients $(22.2 \%$; 95\% CI, 12.5 to 36.3$)$ at week 2,14 patients $(31.1 \%$; $95 \% \mathrm{CI}, 19.5$ to 45.7$)$ at week 4 , and 20 patients $(44.4 \%$; $95 \%$ CI, 30.9 to 58.8$)$ at weeks 6 and 8 . Two patients at week 2 and 6 patients at weeks 4 and 6 were imputed as nonresponders/nonremitters, respectively.

\section{Changes in secondary clinical outcomes}

In the overall study population, symptom severity was decreased, as indicated by a LS mean [SE] change from baseline at week 8 in the CGI-S of -1.7 [0.16] (95\% CI, -2.1 to $-1.4 ; p<0.0001$ ) (Fig. 4A), though the significance level for all secondary outcomes was not adjusted for pre-planned multiple comparisons. Consistent with this, the CGI-I score also indicated improvement following 8 weeks of pimavanserin treatment (LS mean [SE], 2.0 [0.16]; 95\% CI, 1.7 to 2.3) (Fig. 4B). Reductions were also observed for both monotherapy and adjunctive therapy (Table 2).

Patients also reported an improvement in sleep quality. LS mean [SE] change from baseline in GS quality (SCOPA-GS) at week 8 was -1.0 [0.23] (95\% CI, -1.5 to $-0.6 ; p<0.0001$ ) (Fig. 5A). Both NS (SCOPA-NS; LS mean [SE], -2.1 [0.48], 95\% CI, -3.1 to $-1.1 ; p<0.0001$ ) (Fig. 5B) and DS (SCOPA-DS; LS mean [SE], -2.2 [0.33], 95\% $\mathrm{CI},-2.8$ to $-1.5 ; p<0.0001$ ) (Fig. $5 \mathrm{C}$ ) were also improved at week 8 . Similarly, pimavanserin treatment improved caregivers' perception of patients' quality of life, as indicated by an increase from baseline in EQ-5D-5L-VAS score at week 8 (LS mean [SE], 7.0 [2.46]; 95\% CI, 2.1 to $12.0 ; p=0.0068$ ) (Fig. 6).

\section{Safety}

The safety analyses included 47 patients. Twentyone patients experienced 42 TEAEs, with most in the system organ classes (SOC) of gastrointestinal 
A

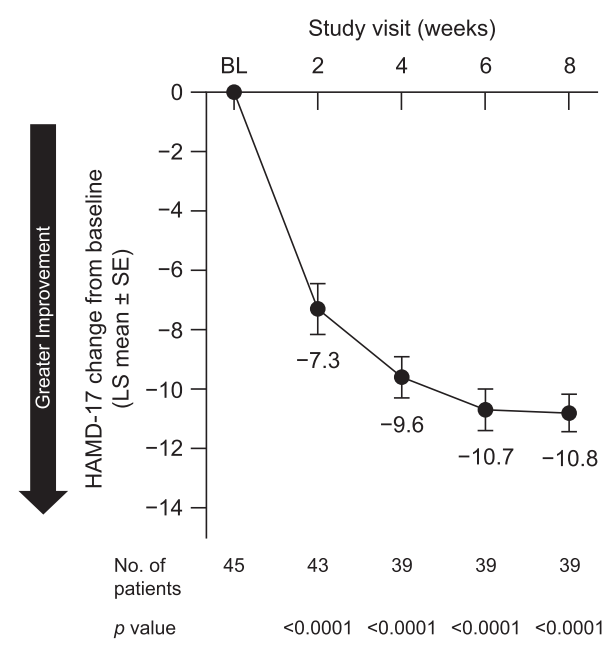

B Individual HAMD-17 Item Scores at Week 8

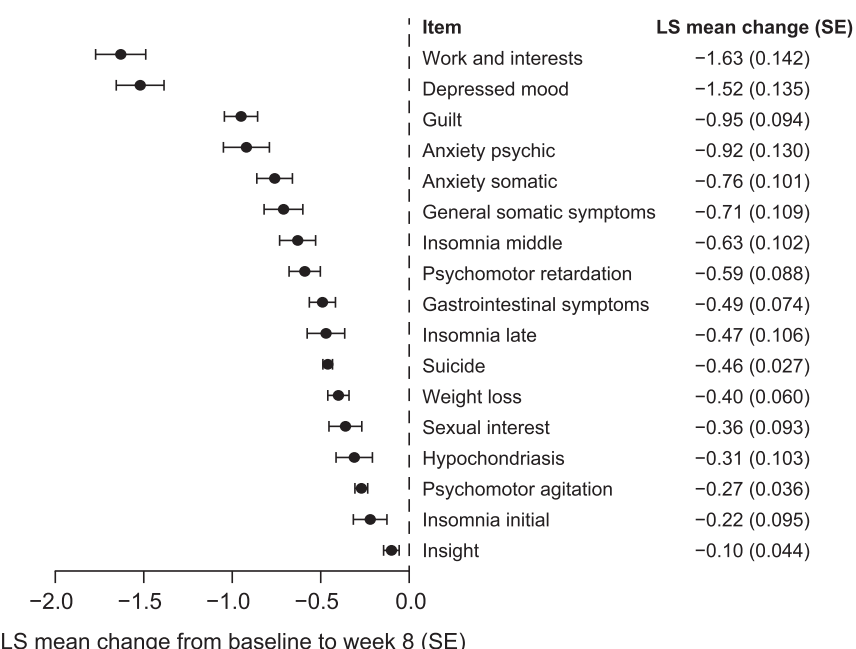

Fig. 2. Change from baseline in HAMD-17 (A) total score over 8 weeks and (B) individual item scores at week 8 in all patients included in efficacy analyses. BL, baseline; HAMD-17, Hamilton Depression Scale-17-item version; LS, least squares; SE, standard error.

A



B

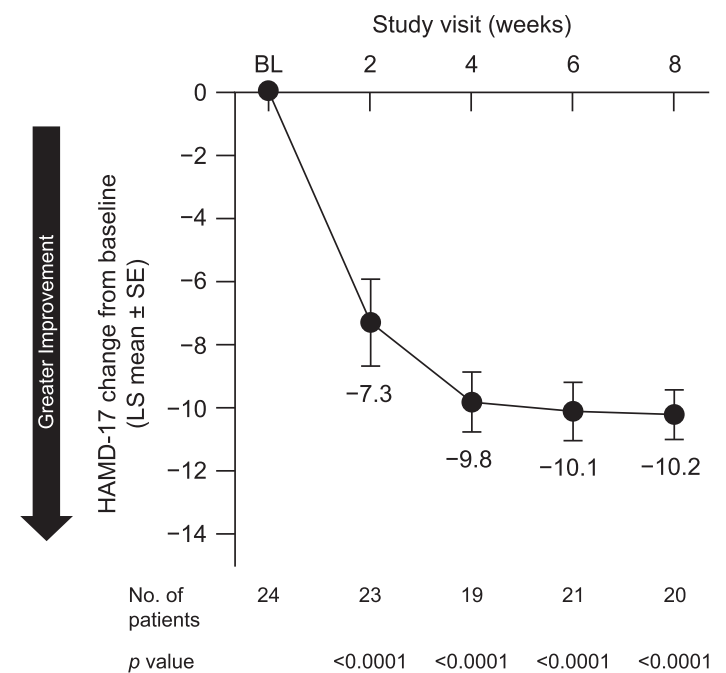

Fig. 3. Change in HAMD-17 from baseline by treatment with pimavanserin as (A) monotherapy and (B) adjunctive therapy. BL, baseline; HAMD-17, Hamilton Depression Scale-17-item version; LS, least squares; SE, standard error.

$(n=7,14.9 \%)$ and psychiatric $(n=7,14.9 \%)$. The most common events by preferred term, across all SOC, were fall $(n=4,8.5 \%)$, nausea $(n=3,6.4 \%)$, diarrhea $(n=2,4.3 \%)$, edema $(n=2,4.3 \%)$, skin abrasion $(n=2,4.3 \%)$, and urinary tract infection $(n=2$, $4.3 \%$ ) (Table 3). Twelve events in 8 patients were considered to be related to treatment. One serious
TEAE of colitis was reported and was not considered to be related to treatment. No deaths were reported.

No change from baseline was observed in cognitive function, as measured by the MMSE, at either week 4 (LS mean [SE], 0.3 [0.26]; $p=0.2078$ ) or week 8 (LS mean [SE], 0.4 [0.25]; $p=0.1266$ ). Furthermore, no clinically significant changes induced by 

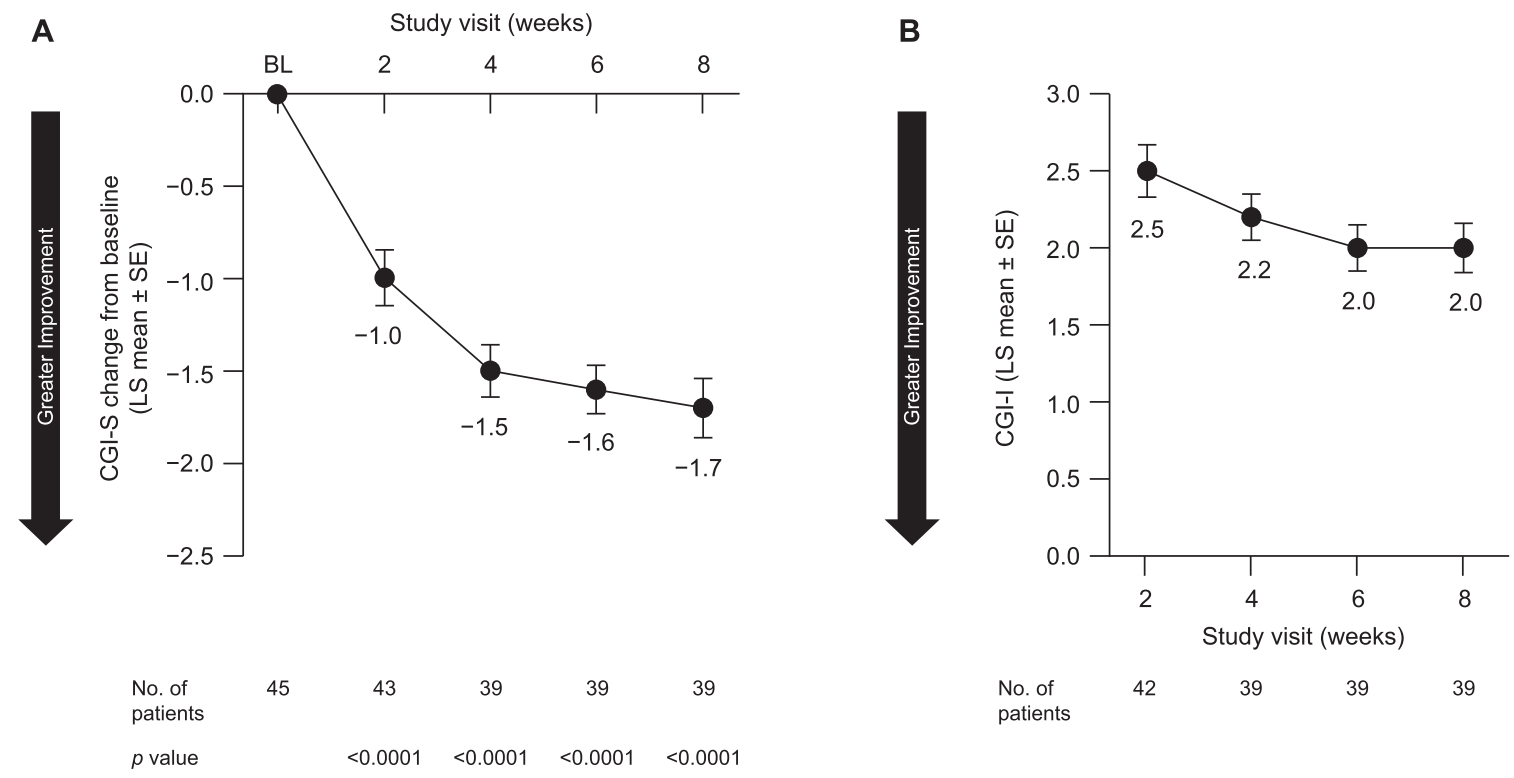

Fig. 4. Impact on (A) CGI-S and (B) CGI-I scores over 8 weeks in all patients included in efficacy analyses. BL, baseline; CGI-I, Clinical Global Impression-Improvement; CGI-S, Clinical Global Impression-Severity; LS, least squares; SE, standard error.

Table 2

Change in CGI-S and CGI-I scores over 8 weeks of treatment with pimavanserin as monotherapy vs adjunctive therapy

\begin{tabular}{lcccc}
\hline & & Monotherapy & Adjunctive Therapy \\
\hline CGI-S & $\mathrm{n}$ & $\begin{array}{c}\text { Change from baseline } \\
\text { LS mean (SE) }\end{array}$ & $\mathrm{n}$ & $\begin{array}{c}\text { Change from baseline } \\
\text { LS mean (SE) }\end{array}$ \\
Week 2 & 20 & $-1.1(0.18)$ & 23 & $-0.9(0.23)$ \\
Week 4 & 20 & $-1.5(0.19)$ & 19 & $-1.5(0.21)$ \\
Week 6 & 18 & $-1.7(0.20)$ & 21 & $-1.5(0.18)$ \\
Week 8 & 19 & $-1.9(0.21)$ & 20 & $-1.6(0.22)$ \\
CGI-I & $\mathrm{n}$ & LS mean (SE) & $\mathrm{n}$ & LS mean (SE) \\
Week 2 & 19 & $2.4(0.21)$ & 23 & $2.6(0.26)$ \\
Week 4 & 20 & $2.1(0.20)$ & 19 & $2.3(0.23)$ \\
Week 6 & 18 & $1.9(0.19)$ & 21 & $2.2(0.22)$ \\
Week 8 & 19 & $1.8(0.20)$ & 20 & $2.2(0.25)$ \\
\hline
\end{tabular}

Clinicians rated the severity of the patient's depression and improvement of symptoms from baseline on a scale of 1-7, with lower scores indicating improvement. CGI-I, Clinical Global Impression-Improvement; CGI-S, Clinical Global Impression-Severity; LS, least squares; SE, standard error.

pimavanserin regarding cardiovascular safety were reported. No patients exhibited a QTcF of $>500 \mathrm{~ms}$ or a change from baseline of $>60 \mathrm{~ms}$ at any point during the study. At baseline, the mean (SE) QTcF interval was 411.0 (3.01) ms. After 8 weeks of pimavanserin treatment, the mean (SE) QTcF interval was 416.8 (3.33) ms, with a mean change from baseline of 8.4 (2.26) $\mathrm{ms}$ (range, -18 to 39 ).

In the safety analysis, no negative effect of pimavanserin on motor function was observed. In contrast, we observed a statistically significant improvement in motor function, as measured by the MDS-UPDRS III, at both week $4(p=0.0023)$ and week 8 ( $p=0.0007)$ (Fig. 7).

\section{DISCUSSION}

These data from a single-arm, open-label 8week study suggest that pimavanserin is associated with early and sustained improvement of depression symptoms in patients with PD. By week 8 , $60.0 \%$ of patients showed $\geq 50 \%$ improvement in symptoms on the HAMD-17, and $44.4 \%$ of patients reached remission (HAMD-17 score $\leq 7$ ) at week 6 , which was sustained through week 8 . Further, similar improvements were seen in patients administered pimavanserin as monotherapy and patients administered pimavanserin as adjunctive therapy with an SSRI or SNRI. Treatment was associated with 
A

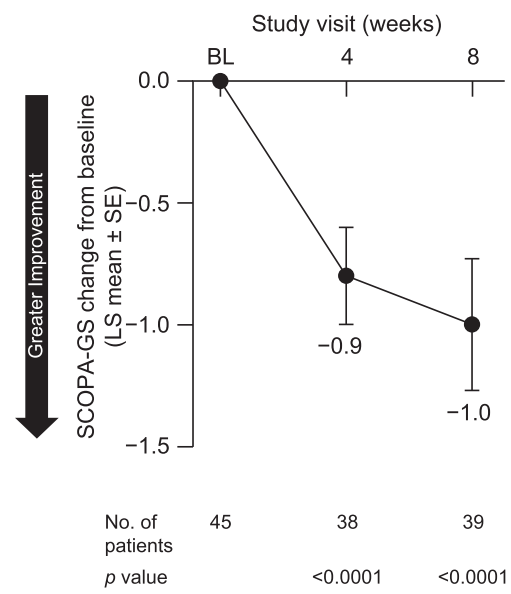

B

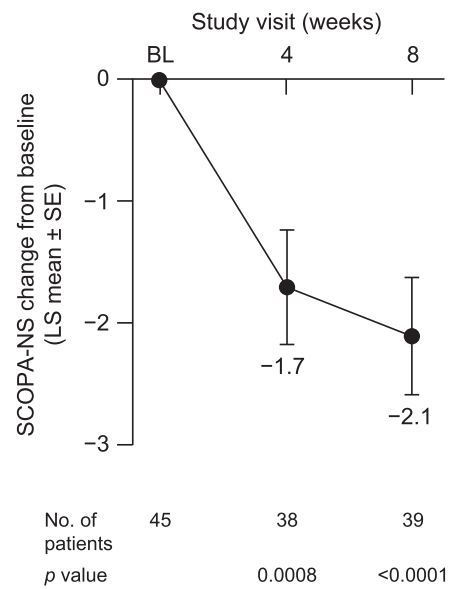

C

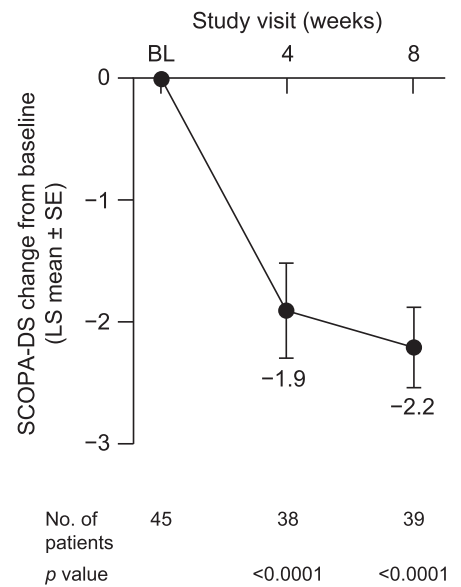

Fig. 5. Change from baseline to week 8 in (A) SCOPA-GS, (B) SCOPA-NS, and (C) SCOPA-DS in all patients included in efficacy analyses. BL, baseline; LS, least squares; SCOPA-DS, Scale for Outcomes in Parkinson's Disease-daytime sleepiness; SCOPA-GS, Scale for Outcomes in Parkinson's Disease-global sleep; SCOPA-NS, Scale for Outcomes in Parkinson's Disease-nighttime sleep; SE, standard error.
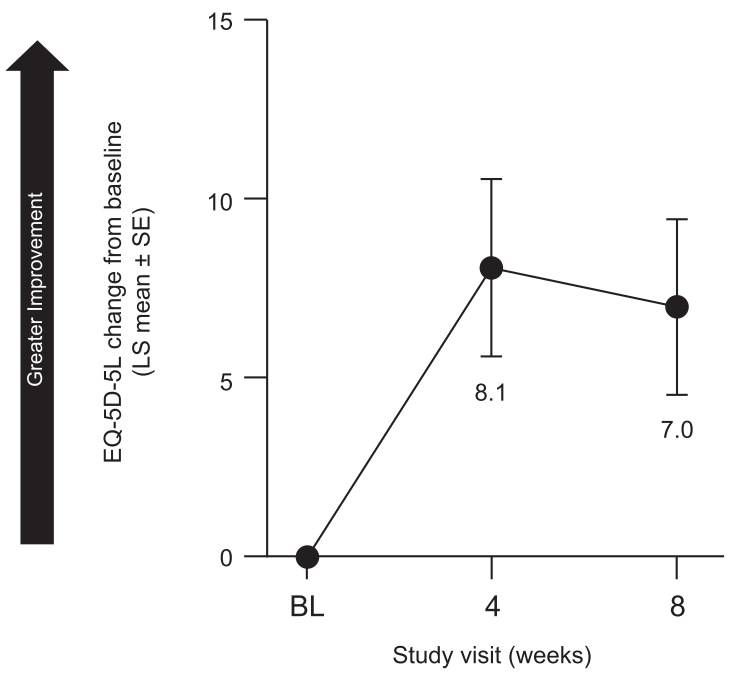

$\begin{array}{lccc}\begin{array}{l}\text { No. of } \\ \text { patients }\end{array} & 45 & 38 & 38 \\ p \text { value } & & 0.0024 & 0.0068\end{array}$

Fig. 6. Change from baseline to week 8 in EQ-5D-5L-VAS in all patients included in efficacy analyses. BL, baseline; EQ-5D5L-VAS, EuroQol-5 Dimensions-5 Levels version 1 visual analog scale; LS, least squares; SE, standard error.

improvements in global assessments of disease severity, quality of sleep, and overall quality of life.

Few studies evaluating currently approved antidepressants for the treatment of PD patients with depression have been conducted, and these studies have shown no or marginal improvement of depressive symptoms [4, 7]. Importantly, antidepres-

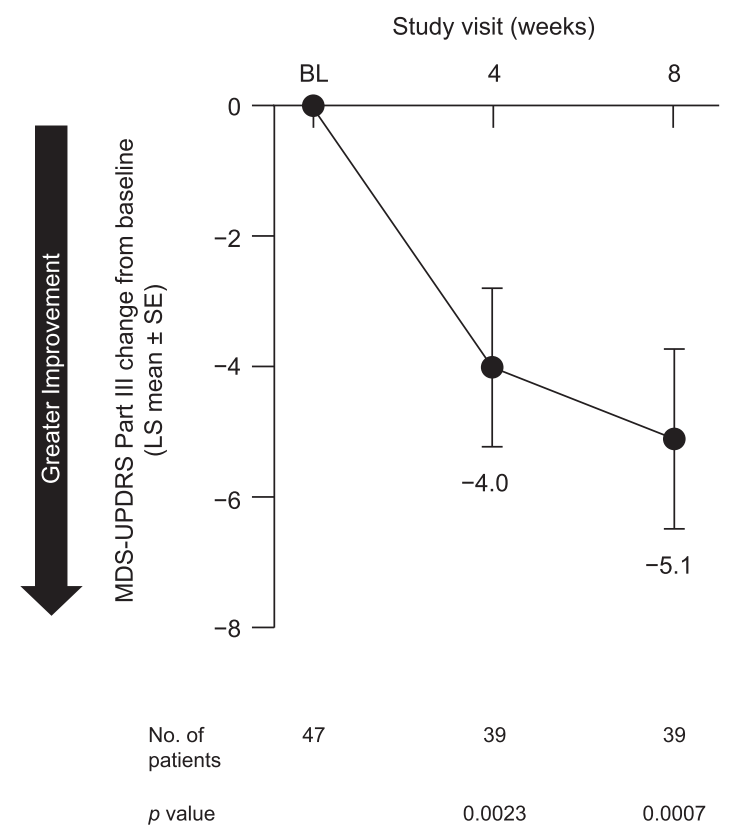

Fig. 7. Change from baseline to week 8 in MDS-UPDRS Part III in all patients included in safety analysis. BL, baseline; LS, least squares; MDS-UPDRS Part III, Movement Disorder Society (MDS)-sponsored revision of the Unified Parkinson's Disease Rating Scale (UPDRS) Part III; SE, standard error.

sant treatments may take several weeks to achieve efficacy for those who do experience some therapeutic benefits [27]. The latency period between the initiation treatment and the onset of the therapeutic benefit exacerbates the public health burden and increases risks for suicide or self-harm [27]. In 
Table 3

Summary of treatment-emergent adverse events

\begin{tabular}{|c|c|}
\hline $\begin{array}{l}\text { Patients with Treatment-Emergent } \\
\text { Adverse Events (TEAEs), n (\%) }\end{array}$ & $\begin{array}{l}\text { Safety } \\
\text { Population } \\
(\mathrm{N}=47)\end{array}$ \\
\hline Any TEAE & $21(44.7)$ \\
\hline Any serious TEAE & $1(2.1)$ \\
\hline \multicolumn{2}{|l|}{ TEAEs by MedDRA preferred term } \\
\hline Fall & $4(8.5)$ \\
\hline Nausea & $3(6.4)$ \\
\hline Diarrhea & $2(4.3)$ \\
\hline Edema & $2(4.3)$ \\
\hline Skin abrasion & $2(4.3)$ \\
\hline Urinary tract infection & $2(4.3)$ \\
\hline Abdominal pain & $1(2.1)$ \\
\hline Abnormal dreams & $1(2.1)$ \\
\hline Blood glucose increased & $1(2.1)$ \\
\hline Blood pressure increased & $1(2.1)$ \\
\hline Colitis & $1(2.1)$ \\
\hline Constipation & $1(2.1)$ \\
\hline Contusion & $1(2.1)$ \\
\hline Dizziness & $1(2.1)$ \\
\hline Gastritis & $1(2.1)$ \\
\hline Gout & $1(2.1)$ \\
\hline Hallucination, auditory & $1(2.1)$ \\
\hline Hallucination, visual & $1(2.1)$ \\
\hline Hypertonia & $1(2.1)$ \\
\hline Hypothyroidism & $1(2.1)$ \\
\hline Illusion & $1(2.1)$ \\
\hline Insomnia & $1(2.1)$ \\
\hline Laceration & $1(2.1)$ \\
\hline Mental impairment & $1(2.1)$ \\
\hline Muscle strain & $1(2.1)$ \\
\hline Non-cardiac chest pain & $1(2.1)$ \\
\hline Palpitations & $1(2.1)$ \\
\hline Peripheral swelling & $1(2.1)$ \\
\hline Presyncope & $1(2.1)$ \\
\hline $\begin{array}{l}\text { Rapid eye movement sleep behavior } \\
\text { disorder }\end{array}$ & $1(2.1)$ \\
\hline Suicidal ideation & $1(2.1)$ \\
\hline Supraventricular extrasystoles & $1(2.1)$ \\
\hline Vomiting & $1(2.1)$ \\
\hline
\end{tabular}

MedDRA, Medical Dictionary for Regulatory Activities, Version 20.0 .

this study, improvements in depressive symptoms emerged by the first study visit, 2 weeks after the start of treatment, with continued improvement throughout the 8-week study. These results suggest that pimavanserin has the potential for rapid antidepressant efficacy in patients with PD.

Management of depression in patients with PD is a critical component of treatment that influences quality of life [3]. Although the HAMD-17 was not developed primarily for use in PD patients and includes some questions about symptoms that may overlap with the symptoms of PD [18], it has good sensitivity in PD populations [28-30]. Patients in in this study exhibited stable symptoms of PD but strong improvements on the HAMD-17 following pimavanserin treatment. Consistent with this, patients also experienced improvements on both physicianreported and patient-reported outcomes, such as the CGI and the SCOPA.

Pimavanserin was well tolerated and safety outcomes were consistent with those from other studies of pimavanserin treatment in patients with PD [31, 32]. The most common TEAEs were fall, nausea, diarrhea, edema, skin abrasion, and urinary tract infection. Interestingly, an improvement in motor function was observed, as indicated by an LS mean reduction from baseline to week 8 of 5.1 on the MDSUPDRS Part III.

Although the pimavanserin treatment results compared with baseline were robust and were consistent with improvements seen in the recent placebocontrolled trial showing efficacy for pimavanserin as an adjunctive treatment in MDD [15], this study was an open-label, single-arm design trial. Because eligible patients were required to have depressive symptoms based on HAMD-17 score, regression to the mean may have contributed to improvements observed on this measure, although the consistent improvement observed across measures supports the strength of the results. The small sample size and lack of a placebo comparator group in this study limit the generalizability of the results. Placebocontrolled studies will be needed to further determine the efficacy of pimavanserin in treating depression in patients with PD.

Patients with PD represent a clinical population with an unmet need for effective pharmacotherapies to improve depressive symptoms. Overall, these results suggest that pimavanserin treatment may be a potential therapeutic opportunity for further evaluation for improvement of depression in patients with PD.

\section{ACKNOWLEDGMENTS}

The authors gratefully acknowledge the patients and clinicians who participated in this study. ACADIA Pharmaceuticals Inc. (San Diego, CA, USA) provided funding for medical writing and editorial support in the development of this manuscript. Meghan Jones, PhD (Ashfield Healthcare Communications, Middletown, CT) provided writing support based on input from authors, and Mary Kacillas and Dena McWain (Ashfield Healthcare Communications) copyedited and styled the manuscript per journal requirements. 


\section{Disclosures}

$\mathrm{DD}, \mathrm{BC}, \mathrm{LJ}, \mathrm{RN}, \mathrm{VA}$ : are employees of and hold stock and/or stock options in ACADIA Pharmaceuticals Inc.

JCN: was an employee of ACADIA Pharmaceuticals Inc. at the time of this study.

GA: has received research support from Accera, Allergan, Axovant, Eisai, Genentech, Intra-Cellular, Janssen, Lundbeck, Neurim, Neurotrope, Novartis, Otsuka, Roche, Suven, and TransTech and has served on the speakers bureau or as a consultant for ACADIA, Alkermes, Allergan, Avanir, Janssen, Lundbeck, Merck, Nestlé, Otsuka, Sunovion, Takeda, and Vanda.

JLA: has received research support from Abbott, AbbVie, ACADIA, Biogen, Boston Scientific, Denali, Impax, NeuroDerm, Sunovion, and Theravance and has received honoraria from Abbott, AbbVie, Acorda, Adamas, Allergan, Boston Scientific, Medtronic, Teva, and US WorldMeds.

$\mathrm{MC}$ is a consultant to ACADIA, Kyowa Kirin, Sunovion, and Reviva.

\section{Data sharing statement}

Data available on request from authors: The data that support the findings of this study are available from the corresponding author upon reasonable request.

\section{SUPPLEMENTARY MATERIAL}

The supplementary material is available in the electronic version of this article: https://dx. doi.org/10.3233/JPD-202058.

\section{REFERENCES}

[1] Marsh L (2013) Depression and Parkinson's disease: Current knowledge. Curr Neurol Neurosci Rep 13, 409.

[2] Slaughter JR, Slaughter KA, Nichols D, Holmes SE, Martens MP (2001) Prevalence, clinical manifestations, etiology, and treatment of depression in Parkinson's disease. $J$ Neuropsychiatry Clin Neurosci 13, 187-196.

[3] Menza M, Dobkin RD, Marin H, Mark MH, Gara M, Buyske S, Bienfait K, Dicke A (2009) The impact of treatment of depression on quality of life, disability and relapse in patients with Parkinson's disease. Mov Disord 24, 13251332.

[4] Liu J, Dong J, Wang L, Su Y, Yan P, Sun S (2013) Comparative efficacy and acceptability of antidepressants in Parkinson's disease: A network meta-analysis. PLoS One 8, e76651.
[5] Rocha FL, Murad MG, Stumpf BP, Hara C, Fuzikawa CJJop (2013) Antidepressants for depression in Parkinson's disease: Systematic review and meta-analysis. 27, 417-423.

[6] Bomasang-Layno E, Fadlon I, Murray AN, Himelhoch S (2015) Antidepressive treatments for Parkinson's disease: A systematic review and meta-analysis. Parkinsonism Relat Disord 21, 833-842; discussion 833.

[7] Weintraub D, Morales KH, Moberg PJ, Bilker WB, Balderston C, Duda JE, Katz IR, Stern MB (2005) Antidepressant studies in Parkinson's disease: A review and meta-analysis. Mov Disord 20, 1161-1169.

[8] Prange S, Metereau E, Thobois S (2019) Structural imaging in Parkinson's disease: New developments. Curr Neurol Neurosci Rep 19, 50.

[9] Thobois S, Prange S, Sgambato-Faure V, Tremblay L, Broussolle E (2017) Imaging the etiology of apathy, anxiety, and depression in Parkinson's disease: Implication for treatment. Curr Neurol Neurosci Rep 17, 76.

[10] Takahashi M, Tabu H, Ozaki A, Hamano T, Takeshima T, group Rs (2019) Antidepressants for depression, apathy, and gait instability in Parkinson's disease: A multicenter randomized study. Intern Med 58, 361-368.

[11] Otte C, Gold SM, Penninx BW, Pariante CM, Etkin A, Fava M, Mohr DC, Schatzberg AF (2016) Major depressive disorder. Nat Rev Dis Primers 2, 16065.

[12] Celada P, Puig M, Amargós-Bosch M, Adell A, Artigas F (2004) The therapeutic role of 5-HT1A and 5-HT2A receptors in depression. J Psychiatry Neurosci 29, 252-265.

[13] Fornaro M, Fusco A, Anastasia A, Cattaneo CI, De Berardis D (2019) Brexpiprazole for treatment-resistant major depressive disorder. Expert Opin Pharmacother 16, 1925-1933.

[14] Hacksell U, Burstein ES, McFarland K, Mills RG, Williams H (2014) On the discovery and development of pimavanserin: A novel drug candidate for Parkinson's psychosis. Neurochem Res 39, 2008-2017.

[15] Fava M, Dirks B, Freeman MP, Papakostas GI, Shelton RC, Thase ME, Trivedi MH, Liu K, Stankovic S (2019) A Phase 2, randomized, double-Blind, placebo-controlled study of adjunctive pimavanserin in patients with major depressive disorder and an inadequate response to therapy (CLARITY). J Clin Psychiatry 80, 19m12928.

[16] Marsh L, McDonald WM, Cummings J, Ravina B, NINDS/NIMH Work Group on Depression and Parkinson's Disease (2006) Provisional diagnostic criteria for depression in Parkinson's disease: Report of an NINDS/NIMH Work Group. Mov Disord 21, 148-158.

[17] Folstein MF, Folstein SE, McHugh PR (1975) "Mini-mental state". A practical method for grading the cognitive state of patients for the clinician. J Psychiatr Res 12, 189-198.

[18] Hamilton M (1960) A rating scale for depression. J Neurol Neurosurg Psychiatry 23, 56-62.

[19] Zimmerman M, Martinez JH, Young D, Chelminski I, Dalrymple K (2013) Severity classification on the Hamilton Depression Rating Scale. J Affect Disord 150, 384-388.

[20] Guy W (1976) Clinical Global Impressions. In: ECDEU Assessment Manual for Psychopharmacology - Revised (DHEW publication number ADM 76-338), 218-222.

[21] Marinus J, Visser M, van Hilten JJ, Lammers GJ, Stiggelbout AM (2003) Assessment of sleep and sleepiness in Parkinson disease. Sleep 26, 1049-1054.

[22] Herdman M, Gudex C, Lloyd A, Janssen M, Kind P, Parkin D, Bonsel G, Badia X (2011) Development and preliminary testing of the new five-level version of EQ-5D (EQ-5D-5L). Qual Life Res 20, 1727-1736. 
[23] Posner K, Brown GK, Stanley B, Brent DA, Yershova KV, Oquendo MA, Currier GW, Melvin GA, Greenhill L, Shen S, Mann JJ (2011) The Columbia-Suicide Severity Rating Scale: Initial validity and internal consistency findings from three multisite studies with adolescents and adults. $\mathrm{Am} \mathrm{J}$ Psychiatry 168, 1266-1277.

[24] Fava M (2003) Diagnosis and definition of treatmentresistant depression. Biol Psychiatry 53, 649-659.

[25] Fava M, Davidson KG (1996) Definition and epidemiology of treatment-resistant depression. Psychiatr Clin North Am 19, 179-200.

[26] Goetz CG, Fahn S, Martinez-Martin P, Poewe W, Sampaio C, Stebbins GT, Stern MB, Tilley BC, Dodel R, Dubois B, Holloway R, Jankovic J, Kulisevsky J, Lang AE, Lees A, Leurgans S, LeWitt PA, Nyenhuis D, Olanow CW, Rascol O, Schrag A, Teresi JA, Van Hilten JJ, LaPelle N (2007) Movement Disorder Society-sponsored revision of the Unified Parkinson's Disease Rating Scale (MDS-UPDRS): Process, format, and clinimetric testing plan. Mov Disord 22, 41-47.

[27] Machado-Vieira R, Henter ID, Zarate CA Jr (2017) New targets for rapid antidepressant action. Prog Neurobiol 152, 21-37.

[28] Schrag A, Barone P, Brown RG, Leentjens AF, McDonald WM, Starkstein S, Weintraub D, Poewe W, Rascol O, Sampaio C, Stebbins GT, Goetz CG (2007) Depression rating scales in Parkinson's disease: Critique and recommendations. Mov Disord 22, 1077-1092.
[29] Richard IH, McDermott MP, Kurlan R, Lyness JM, Como PG, Pearson N, Factor SA, Juncos J, Serrano Ramos C, Brodsky M, Manning C, Marsh L, Shulman L, Fernandez HH, Black KJ, Panisset M, Christine CW, Jiang W, Singer C, Horn S, Pfeiffer R, Rottenberg D, Slevin J, Elmer L, Press D, Hyson HC, McDonald W, Group S-PS (2012) A randomized, double-blind, placebo-controlled trial of antidepressants in Parkinson disease. Neurology 78, 12291236.

[30] Menza M, Dobkin RD, Marin H, Mark MH, Gara M, Buyske S, Bienfait K, Dicke A (2009) A controlled trial of antidepressants in patients with Parkinson disease and depression. Neurology 72, 886-892.

[31] Cummings J, Isaacson S, Mills R, Williams H, Chi-Burris K, Corbett A, Dhall R, Ballard C (2014) Pimavanserin for patients with Parkinson's disease psychosis: A randomised, placebo-controlled phase 3 trial. Lancet 383, 533-540.

[32] Meltzer HY, Mills R, Revell S, Williams H, Johnson A, Bahr D, Friedman JH (2010) Pimavanserin, a serotonin(2A) receptor inverse agonist, for the treatment of parkinson's disease psychosis. Neuropsychopharmacology 35, 881-892. 